\title{
The Berger-Shaw Theorem for Cyclic Subnormal Operators
}

\author{
NATHAN S. FELDMAN
}

\begin{abstract}
This work gives a sharp form of the Berger-Shaw Theorem for cyclic subnormal operators. That is, if $S$ is a cyclic subnormal operator, then $S$ has trace class selfcommutator and the trace equals $(1 / \pi) \operatorname{Area}\left[\sigma(S)-\sigma_{e}(S)\right]$. It also characterizes those functions $f$ such that $f(S)$ has trace class self-commutator and computes the trace as the Dirichlet integral of $\hat{f}$ on the set of analytic bounded point evaluations for $S$. The technique used also gives some partial results for rationally cyclic subnormal operators.
\end{abstract}

Introduction. For an operator $T$ the self-commutator of $T$ is $\left[T^{*}, T\right]=$ $T^{*} T-T T^{*}$. The Berger-Shaw Theorem (see [4], p. 152) implies that every rationally cyclic hyponormal operator $T$ has trace class self-commutator and $\operatorname{tr}\left[T^{*}, T\right] \leq(1 / \pi)$ Area $[\sigma(T)]$. This work considers the case of a pure cyclic subnormal operator $S$ and gives another proof that $S$ has trace class self-commutator and computes the trace as $\operatorname{tr}\left[S^{*}, S\right]=(1 / \pi)$ Area $\left[\sigma(S)-\sigma_{e}(S)\right]$. It follows that the principal function for a cyclic subnormal operator vanishes a.e. on the essential spectrum. We also characterize the operators in the commutant of $S$ that have trace class self-commutator. The main tools used are Thomson's Theorem on the existence of bounded point evaluations for cyclic subnormal operators and the Intertwining Lemma of Berger and Shaw allowing one to compare the trace of the self-commutators of two hyponormal operators.

If $\mu$ is a positive compactly supported regular Borel measure in $\mathbf{C}$, then let $P^{2}(\mu)$ denote the closure of the (analytic) polynomials in $L^{2}(\mu)$. If $S_{\mu}=M_{z}$ on $P^{2}(\mu)$, then $S_{\mu}$ is a cyclic subnormal operator. Furthermore, every cyclic subnormal operator can be represented in this form for some measure $\mu$ (see [4], p. 52). If $f \in P^{2}(\mu) \cap L^{\infty}(\mu)$, then $f\left(S_{\mu}\right)$ denotes the multiplication operator on $P^{2}(\mu)$ with symbol $f$.

For a given measure $\mu$ let $G=\operatorname{abpe}(\mu)$ be the set of analytic bounded point evaluations for $P^{2}(\mu)$. If $S_{\mu}$ is pure, then $G=\sigma\left(S_{\mu}\right)-\sigma_{e}\left(S_{\mu}\right)$. For each $\lambda$ in 
$G$, the reproducing kernel for the point $\lambda$ is a function $k_{\lambda}$ in $P^{2}(\mu)$ such that $p(\lambda)=\left\langle p, k_{\lambda}\right\rangle$ for every polynomial $p$. Also for $f$ in $P^{2}(\mu)$, let $\hat{f}(\lambda)=\left\langle f, k_{\lambda}\right\rangle$ be the analytic extension of $f$ to $G$.

We shall use Thomson's Theorem, see [4] or [12], on the plentiful supply of analytic bounded point evaluations to prove that $f\left(S_{\mu}\right)$ has trace class selfcommutator if and only if $\hat{f}$ is in the Dirichlet space on $G$, that is $\int_{G}\left|\hat{f}^{\prime}\right|^{2} d A<\infty$. Furthermore, we shall show that

$$
\operatorname{tr}\left[f\left(S_{\mu}\right)^{*}, f\left(S_{\mu}\right)\right]=\frac{1}{\pi} \int_{G}\left|\hat{f}^{\prime}\right|^{2} d A
$$

For various Hilbert spaces $\mathcal{H}$ of analytic functions on a bounded region $G$ it has been shown that for $f \in H^{\infty}(G)$ the operator $T_{f}$, of multiplication by $f$ on $\mathcal{H}$, satisfies $\operatorname{tr}\left[T_{f}^{*}, T_{f}\right]=(1 / \pi) \int_{G}\left|f^{\prime}\right|^{2} d A$. For example this holds if $\mathcal{H}$ is a weighted Bergman space on $G$. For the unweighted Bergman space this is due to Berger and Shaw; see [2] for the weighted case. Recently, Aleman [1] has shown the above equality to be true when $\mathcal{H}$ is the Hardy space of an arbitrary bounded region.

1. Cyclic subnormal operators. We now state and prove the main theorem.

Main Theorem. If $S_{\mu}=M_{z}$ on $P^{2}(\mu)$ is a pure cyclic subnormal operator, $G=$ abpe $(\mu)$ and $f \in P^{2}(\mu) \cap L^{\infty}(\mu)$, then $\left[f\left(S_{\mu}\right)^{*}, f\left(S_{\mu}\right)\right]$ is trace class if and only if $\hat{f}$ is in the Dirichlet space on $G$. In that case,

$$
\operatorname{tr}\left[f\left(S_{\mu}\right)^{*}, f\left(S_{\mu}\right)\right]=\frac{1}{\pi} \int_{G}\left|\hat{f}^{\prime}\right|^{2} d A .
$$

We shall state the Intertwining Lemma for completeness. See [3], p. 73, for a nice proof.

Intertwining Lemma. Let $S$ and $T$ be hyponormal operators on $\mathcal{H}$ and $\mathcal{K}$ respectively. If there exists a one-to-one Hilbert-Schmidt operator $W: \mathcal{H} \rightarrow \mathcal{K}$ with dense range satisfying $W S=T W$, then $\operatorname{tr}\left[T^{*}, T\right] \leq \operatorname{tr}\left[S^{*}, S\right]$.

In what follows, $H^{\infty}(G)$ is the algebra of bounded analytic functions on the region $G$ and $H^{2}(G)$ is the Hardy space. Also, let $S_{G}=M_{z}$ on $H^{2}(G)$ and $f\left(S_{G}\right)=M_{f}$ on $H^{2}(G)$.

Lemma 1.1. If $G$ is a bounded simply connected region and $f \in H^{\infty}(G)$, then the multiplication operator $f\left(S_{G}\right)$ has trace class self-commutator if and only if $f$ is in the Dirichlet space on $G$. Also, $\operatorname{tr}\left[f\left(S_{G}\right)^{*}, f\left(S_{G}\right)\right]=(1 / \pi) \int_{G}\left|f^{\prime}\right|^{2} d A$. 
Proof. If $G=\mathbf{D}$, then this is an easy computation involving the Fourier coefficients of $f$. In general, let $\varphi: \mathbf{D} \rightarrow G$ be a Riemann map. If $f \in$ $H^{\infty}(G)$, then the operator $f\left(S_{G}\right)$ is unitarily equivalent to $(f \circ \varphi)\left(S_{D}\right)$, thus with a simple change of variables we see that the general case can be reduced to the unit disk, $\mathbf{D}$.

Let $G$ be any bounded region in $\mathbf{C}$. We say that a Hilbert space $\mathcal{H}$ is a Hilbert space of analytic functions on $G$ if $\mathcal{H}$ is a vector subspace of $\operatorname{Hol}(G)$, the Frechet space of Holomorphic functions on $G$, such that the inclusion of $\mathcal{H}$ into $\operatorname{Hol}(G)$ is continuous. The following Lemma shall be proved after the Theorem.

Lemma 1.2. If $G$ is any bounded region, then there exists a sequence of regions $\left\{G_{n}\right\}$ such that for each $n, G_{n}$ is bounded by an analytic Jordan curve, $\operatorname{cl} G_{n} \subseteq G_{n+1} \subseteq G, \bigcup_{n=1}^{\infty} G_{n}$ is dense in $G$ and $\operatorname{Area}\left(G_{n}\right) \rightarrow \operatorname{Area}(G)$ as $n \rightarrow \infty$.

Theorem 1.3. If $\mathcal{H}$ is any Hilbert space of analytic functions on a bounded region $G$ that contains the polynomials and $f \in H^{\infty}(G)$ is such that multiplication by $f, M_{f}$, on $\mathcal{H}$ is a hyponormal operator, then

$$
\operatorname{tr}\left[M_{f}^{*}, M_{f}\right] \geq \frac{1}{\pi} \int_{G}\left|f^{\prime}\right|^{2} d A .
$$

Proof. Let $\left\{G_{n}\right\}$ be as in Lemma 1.2. For a fixed $n$, define $W_{n}: \mathcal{H} \rightarrow$ $H^{2}\left(G_{n}\right)$ as the restriction operator, since $G_{n}$ is compactly contained in $G$, this is a well-defined bounded linear operator. Clearly, $W$ is one-to-one, and since $\mathcal{H}$ contians the polynomials and $\partial G_{n}$ is a Jordan curve, $W_{n}$ has dense range. Clearly $W_{n}$ intertwines $S=\left(M_{f}, \mathcal{H}\right)$ and $T=\left(M_{f}, H^{2}\left(G_{n}\right)\right)$ and both are hyponormal operators. We now show that $W_{n}$ is Hilbert-Schmidt.

So, let $\left\{f_{k}\right\}$ be any orthonormal basis for $\mathcal{H}$. Also, let $K_{z}$ be the reproducing kernels in $\mathcal{H}$ for $z \in G$ and fix an integer $n$. Observe that

$$
K_{z}=\sum_{k=1}^{\infty}\left\langle K_{z}, f_{k}\right\rangle f_{k}=\sum_{k=1}^{\infty} \overline{f_{k}(z)} f_{k}
$$

so

$$
\left\|K_{z}\right\|_{\mu}^{2}=\sum_{k=1}^{\infty}\left|f_{k}(z)\right|^{2}
$$

Thus we have

$$
\sum_{k=1}^{\infty}\left\|W_{n} f_{k}\right\|_{H^{2}\left(G_{n}\right)}^{2}=\sum_{k=1}^{\infty} \int_{\partial G_{n}}\left|f_{k}(z)\right|^{2} d \omega_{n}=
$$




$$
\begin{aligned}
& =\int_{\partial G_{n}} \sum_{k=1}^{\infty}\left|f_{k}(z)\right|^{2} d \omega_{n} \\
& =\int_{\partial G_{n}}\left\|K_{z}\right\|_{\mathcal{H}}^{2} d \omega_{n}<\infty .
\end{aligned}
$$

The last integral is finite because the reproducing kernels $\left\{K_{z}: z \in \operatorname{cl} G_{n}\right\}$ form a bounded set in $\mathcal{H}$. Thus $W_{n}$ is Hilbert-Schmidt. The Intertwining Lemma now applies to give that $\operatorname{tr}\left[S^{*}, S\right] \geq \operatorname{tr}\left[T^{*}, T\right]$. But since $G_{n}$ is simply connected, Lemma 1.1 implies that $\operatorname{tr}\left[T^{*}, T\right]=(1 / \pi) \int_{G_{n}}\left|f^{\prime}\right|^{2} d A$. Thus, $\operatorname{tr}\left[S^{*}, S\right] \geq(1 / \pi) \int_{G_{n}}\left|f^{\prime}\right|^{2} d A$ for all $n$. Now, since Area $\left(G_{n}\right) \rightarrow \operatorname{Area}(G)$, letting $n \rightarrow \infty$ the monotone convergence theorem gives the desired conclusion.

Proof of Lemma 1.2. If $G$ is a bounded region, let $\tau: \mathbf{D} \rightarrow G$ be a universal covering map. A fundamental region for $\tau$ is a region $F$ in $\mathbf{D}$ so that $\tau(\operatorname{cl} F \cap \mathbf{D})=G$ and $\tau$ is one-to-one on $F$. Let $F$ be a "Dirichlet region" for $\tau$, that is a particular type of fundamental region that is an intersection of hyperbolic half-planes in $\mathbf{D}$, see [7], p. 52. Since the region $F$ is simply connected, in fact hyperbolically convex, and $\tau$ is one-to-one on $F$, it follows that $\tau(F)$ is a simply connected region contained in $G$. Also, $\tau(F)$ is dense in $G$, since $\tau$ maps $\mathrm{cl} F \cap \mathbf{D}$ onto $G$. Furthermore, since $\partial F$ is contained in a countable number of hyperbolic geodesics, $\partial F$ has area zero. Now, as analytic functions map sets of area zero onto sets of area zero, it follows that $\tau(\partial F)$ has area zero. Since $G-\tau(F) \subseteq \tau(\partial F)$, and this latter set has area zero, it follows that $\operatorname{Area}(G)=\operatorname{Area}(\tau(F))$. Now, let $\varphi: \mathbf{D} \rightarrow \tau(F)$ be a Riemann map. If $G_{n}=\varphi(\{z \in \mathbf{D}:|z|<1-(1 / n)\})$, then $G_{n}$ has the required properties.

Notice that if $G$ is a simply connected region then Lemma 1.2 follows immediately from the Riemann mapping theorem.

Corollary 1.4. If $S_{\mu}$ is a pure cyclic subnormal operator, abpe $(\mu)=\mathbf{D}$ and $f \in P^{2}(\mu) \cap L^{\infty}(\mu)$, then $\operatorname{tr}\left[f\left(S_{\mu}\right)^{*}, f\left(S_{\mu}\right)\right] \geq(1 / \pi) \int_{\mathbf{D}}\left|\hat{f}^{\prime}\right|^{2} d A$, where $\hat{f}$ is the analytic extension of $f$ to $\mathbf{D}$.

Proof. Thomson's Theorem allows us to use the natural map ^ $: P^{2}(\mu) \rightarrow$ $\operatorname{Hol}(\mathbf{D})$ to identify $P^{2}(\mu)$ with a space of analytic functions on $\mathbf{D}$. Also, if $f \in P^{2}(\mu) \cap L^{\infty}(\mu)$, then $f\left(S_{\mu}\right)$ is a subnormal operator. Hence Theorem 1.3 applies.

Lemma 1.5. If $S_{\mu}$ is a pure cyclic subnormal operator, abpe $(\mu)=\mathbf{D}$ and $f$ is analytic on a neighborhood of the closed unit disk, clD, then

$$
\operatorname{tr}\left[f\left(S_{\mu}\right)^{*}, f\left(S_{\mu}\right)\right] \leq \frac{1}{\pi} \int_{\mathbf{D}}\left|f^{\prime}\right|^{2} d A .
$$


Proof. Suppose $f$ is analytic on $\{z:|z|<r\}$ for some $r>1$. Let $G=$ $\left\{z:|z|<r_{0}\right\}$ for some $1<r_{0}<r$. So, $f \in H^{\infty}(G)$. Now, let $S=M_{f}$ on $H^{2}(G)$ and set $T=f\left(S_{\mu}\right)$. Also, define $W: H^{2}(G) \rightarrow P^{2}(\mu)$ by $W h=h \mid$ clD. Clearly, $W S=T W$ and $W$ is one-to-one with dense range. Also, an argument as in Theorem 1.3 shows that $W$ is Hilbert-Schmidt. So, the Intertwining Lemma gives us that $\operatorname{tr}\left[T^{*}, T\right] \leq \operatorname{tr}\left[S^{*}, S\right]=(1 / \pi) \int_{G}\left|f^{\prime}\right|^{2} d A$. The last equality holds by Lemma 1 . Now, letting $r_{0}$ decrease to 1 , gives the result.

\section{Proof of the Main Theorem.}

\Special Case: abpe $(\mu)=\mathbf{D}$.

In this case, let $f \in P^{2}(\mu) \cap L^{\infty}(\mu)$. By Corollary 1.4, it suffices to show that $\operatorname{tr}\left[f\left(S_{\mu}\right)^{*}, f\left(S_{\mu}\right)\right] \leq(1 / \pi) \int_{\mathbf{D}}\left|\hat{f}^{\prime}\right|^{2} d A$. So, suppose that $(1 / \pi) \int_{\mathbf{D}}\left|\hat{f}^{\prime}\right|^{2} d A<\infty$, then $\hat{f} \in D_{1}$, the Dirichlet space on $\mathbf{D}$. Define $f_{j}(z)=\hat{f}\left(r_{j} z\right)$ for some sequence $\left\{r_{j}\right\}$ increasing to 1 . It is easy to check that $f_{j}$ converges to $\hat{f}$ in the Dirichlet norm, thus

$$
\frac{1}{\pi} \int_{\mathbf{D}}\left|f_{j}^{\prime}\right|^{2} d A \rightarrow \frac{1}{\pi} \int_{\mathbf{D}}\left|\hat{f}^{\prime}\right|^{2} d A .
$$

Now, as each $f_{j}$ is analytic on a neighborhood of clD, Corollary 1.4 and Lemma 1.5 give that $\operatorname{tr}\left[f_{j}\left(S_{\mu}\right)^{*}, f_{j}\left(S_{\mu}\right)\right]=(1 / \pi) \int_{\mathbf{D}}\left|f_{j}^{\prime}\right|^{2} d A$. Since $f_{j}\left(S_{\mu}\right)$ is subnormal it follows that $\left[f_{j}\left(S_{\mu}\right)^{*}, f_{j}\left(S_{\mu}\right)\right]$ is positive, hence its trace equals its trace norm. Thus the sequence $\left\{\left[f_{j}\left(S_{\mu}\right)^{*}, f_{j}\left(S_{\mu}\right)\right]: n \geq 1\right\}$ is norm bounded in the space of trace class operators, $\mathcal{B}_{1}$. Since $\mathcal{B}_{1}$ is the dual of the separable space of compact operators $\mathcal{B}_{0}$, there is a weak* convergent subsequence. Let's assume that $\left[f_{j}\left(S_{\mu}\right)^{*}, f_{j}\left(S_{\mu}\right)\right] \rightarrow A$ weak $^{*}$ in $\mathcal{B}_{1}$, that is if $K$ is any compact operator, then $\operatorname{tr}\left(K\left[f_{j}\left(S_{\mu}\right)^{*}, f_{j}\left(S_{\mu}\right)\right]\right) \rightarrow \operatorname{tr}(K A)$. Now, by choosing $K$ to be the rank one operator $h \otimes h$ for some $h \in P^{2}(\mu)$, one sees that since $\left[f_{j}\left(S_{\mu}\right)^{*}, f_{j}\left(S_{\mu}\right)\right]$ is positive for all $n, A$ must be a positive operator in $\mathcal{B}_{1}$.

Claim 1. $\operatorname{tr} A \leq(1 / \pi) \int_{\mathbf{D}}\left|\hat{f}^{\prime}\right|^{2} d A$.

Since $A$ is a positive trace class operator, its trace equals its trace norm. Thus since the norm is lower semi-continuous in the weak* topology, we have

$$
\begin{aligned}
\operatorname{tr} A & =\|A\|_{1} \leq \liminf _{j \rightarrow \infty}\left\|\left[f_{j}\left(S_{\mu}\right)^{*}, f_{j}\left(S_{\mu}\right)\right]\right\|_{1} \\
& =\liminf _{j \rightarrow \infty} \frac{1}{\pi} \int_{\mathbf{D}}\left|f_{j}^{\prime}\right|^{2} d A=\frac{1}{\pi} \int_{\mathbf{D}}|\hat{f}|^{2} d A
\end{aligned}
$$

Observe that the last limit exists because $f_{j}$ converges to $\hat{f}$ in the Dirichlet norm.

Claim 2. $A=\left[f\left(S_{\mu}\right)^{*}, f\left(S_{\mu}\right)\right]$. 
If $h \in P^{2}(\mu)$, then by considering rank one operators, we have $\left\langle\left[f_{j}\left(S_{\mu}\right)^{*}, f_{j}\left(S_{\mu}\right)\right] h, h\right\rangle \rightarrow\langle A h, h\rangle$. Also, as $f_{j}$ converges pointwise boundedly to $f$, we get that $f_{j} h \rightarrow f h$ in $L^{2}(\mu)$ norm and $\bar{f}_{j} h \rightarrow \bar{f} h$ in $L^{2}(\mu)$ norm. Hence if $P$ is the projection of $L^{2}(\mu)$ onto $P^{2}(\mu)$, then

$$
\begin{gathered}
\left\langle\left[f_{j}\left(S_{\mu}\right)^{*}, f_{j}\left(S_{\mu}\right)\right] h, h\right\rangle=\left\|f_{j}\left(S_{\mu}\right) h\right\|^{2}-\left\|f_{j}\left(S_{\mu}\right)^{*} h\right\|^{2}=\left\|f_{j} h\right\|^{2}-\left\|P\left(\bar{f}_{j} h\right)\right\|^{2} \\
\rightarrow\|f h\|^{2}-\|P(\bar{f} h)\|^{2}=\left\langle\left[f\left(S_{\mu}\right)^{*}, f\left(S_{\mu}\right)\right] h, h\right\rangle
\end{gathered}
$$

Hence we have that for each $h \in P^{2}(\mu),\langle A h, h\rangle=\left\langle\left[f\left(S_{\mu}\right)^{*}, f\left(S_{\mu}\right)\right] h, h\right\rangle$. As both operators are self-adjoint, they must be equal.

Thus, since $\left[f\left(S_{\mu}\right)^{*}, f\left(S_{\mu}\right)\right]=A,\left[f\left(S_{\mu}\right)^{*}, f\left(S_{\mu}\right)\right]$ is trace class, and $\operatorname{tr}\left[f\left(S_{\mu}\right)^{*}, f\left(S_{\mu}\right)\right]=\operatorname{tr} A \leq(1 / \pi) \int_{\mathbf{D}}\left|\hat{f}^{\prime}\right|^{2} d A$. Combining this with Corollary 1.4, gives $\operatorname{tr}\left[f\left(S_{\mu}\right)^{*}, f\left(S_{\mu}\right)\right]=(1 / \pi) \int_{\mathbf{D}}\left|\hat{f}^{\prime}\right|^{2} d A$.

@ General Case: $S_{\mu}$ is pure and $G=\operatorname{abpe}(\mu)$.

It follows from Thomson's Theorem that each component of $G$ corresponds to an irreducible summand of $S_{\mu}$, hence $S_{\mu}$ is a direct sum of irreducible cyclic operators and the self-commutator is therefore also a direct sum of self-commutators. Thus it suffices to consider the case where $S_{\mu}$ is irreducible. In particular $G$ is connected.

If $\tau$ is a Riemann map of $\mathbf{D}$ onto $G$, then there is a measure $\nu$ on clD such that $S_{\nu}$ is pure, abpe $(\nu)=\mathbf{D}, \mu=\nu \circ \tau^{-1}$ and $S_{\mu}$ is unitarily equivalent to $\tau\left(S_{\nu}\right)$ (this strongly uses Thomson's Theorem, see [9]). Further, the unitary is given by composition with $\tau$.

If $f \in P^{2}(\mu) \cap L^{\infty}(\mu)$, then $g=f \circ \tau \in P^{2}(\nu) \cap L^{\infty}(\nu)$ and so it follows that $f\left(S_{\mu}\right) \cong g\left(S_{\nu}\right)$. Thus, $\operatorname{tr}\left[f\left(S_{\mu}\right)^{*}, f\left(S_{\mu}\right)\right]=\operatorname{tr}\left[g\left(S_{\nu}\right)^{*}, g\left(S_{\nu}\right)\right]=(1 / \pi) \int_{\mathbf{D}}\left|\hat{g}^{\prime}\right|^{2} d A$. Now, as $\hat{g}=\hat{f} \circ \tau$, a simple change of variables gives that this last integral equals $(1 / \pi) \int_{G}\left|\hat{f}^{\prime}\right|^{2} d A$. So the proof of the Theorem is complete.

If $S$ is any pure cyclic subnormal operator, represented as $M_{z}$ on $P^{2}(\mu)$, then $\sigma(S)-\sigma_{e}(S)$ is exactly the set of analytic bounded point evaluations for $P^{2}(\mu)$.

Corollary 1.6. If $S$ is any cyclic subnormal operator, then $S$ has trace class self-commutator and $\operatorname{tr}\left[S^{*}, S\right]=(1 / \pi) \operatorname{Area}\left[\sigma(S)-\sigma_{e}(S)\right]$.

If $T$ is any pure hyponormal operator with trace class self-commutator, then the principal function for $T, g_{T}$, is a non-negative function defined on $\sigma(T)$ such that $g_{T}(\lambda)=-i n d(T-\lambda)$ for all $\lambda \notin \sigma_{e}(T)$ and $\operatorname{tr}\left[T^{*}, T\right]=(1 / \pi) \int_{\sigma(T)} g_{T} d A$ (see[3] or [8]). 
Corollary 1.7. If $S$ is a pure cyclic subnormal operator, then the principal function for $S$ vanishes a.e. on $\sigma_{e}(S)$.

Proof. If $g_{S}$ is the principal function for $S$ and $G=\sigma(S)-\sigma_{e}(S)$, then we have

$$
\frac{1}{\pi} \operatorname{Area}(G)=\operatorname{tr}\left[S^{*}, S\right]=\frac{1}{\pi} \int_{\sigma(S)} g_{s} d A=\frac{1}{\pi} \int_{G} 1 d A+\frac{1}{\pi} \int_{\sigma_{e}(S)} g_{S} d A
$$

where we have used that $g_{s}(\lambda)=-i n d(S-\lambda)=1$ on $G$. Thus, since $g_{S}$ is non-negative, we see that $g_{S}=0$ a.e. on $\sigma_{e}(S)$.

2. Examples. The following examples show that it is necessary to leave out the essential spectrum in computing the trace of $\left[S_{\mu}^{*}, S_{\mu}\right]$ and that one does not always have equality in the Berger-Shaw Theorem.

Example 2.1. It's known that there exist Jordan curves with positive area (see [10] or [11]). If $G$ is the simply connected region bounded by such a curve, then there exists a measure $\mu$ on $G$, so that $S_{\mu}$ is an irreducible subnormal operator and $\operatorname{abpe}(\mu)=G$ (see [4], p. 405). Hence $\sigma_{e}\left(S_{\mu}\right)=\partial G$ has positive area.

Lemma 2.2. If $B$ is any disk and $K \subseteq B$ is a nowhere dense compact set, then there exists a sequence $\left\{\Delta_{k}\right\}$ of open disks contained in $B-K$ such that $\Delta_{n}$ and $\Delta_{k}$ have disjoint closures if $n \neq k$, the radii of the disks is summable and the disks cluster precisely on $K$.

By this last statement we mean that if $\left\{z_{k}\right\}$ is any sequence with $z_{k} \in \Delta_{k}$, then the limit points of the sequence $\left\{z_{k}\right\}$ is equal to $K$.

Proof. Since $K$ is nowhere dense, $B-K$ is dense in $B$. Now, choose a sequence $\left\{a_{n}\right\}$ of points $B-K$ that clusters precisely on $K$. One may do this as follows, for each integer $n$, cover $K$ with a finite number of open disks with radius $1 / n$. Since $B-K$ is dense in $B$, one may choose one point in each of these disks. In this way the constructed sequence clusters precisely on $K$. So for each $n, a_{n} \in B-K$ is isolated from the other points of this sequence, hence we may choose a disk $\Delta_{n} \subseteq B-K$ centered at $a_{n}$ so that the disks $\left\{\Delta_{n}\right\}$ have disjoint closures. If we now shrink the disks as necessary to guarantee that their radii are summable, then they will still have disjoint closures. Also, since the radii tend to zero, the disks will cluster at precisely the same points as the sequence $\left\{a_{n}\right\}$.

We now use Lemma 2.2 to give two examples. The first example illustrates that even for pure cyclic subnormal operators the essential spectrum can be a very thick subset of the spectrum. In particular it shows how far the inequality in the Berger-Shaw Theorem can be from an equality.

Example 2.3. If $\varepsilon>0$, then there exists a pure cyclic subnormal operator $S_{\mu}$ such that $\operatorname{tr}\left[S_{\mu}^{*}, S_{\mu}\right]<\varepsilon$ and yet $(1 / \pi) \operatorname{Area}\left[\sigma\left(S_{\mu}\right)\right] \geq 1-\varepsilon$. 
Proof. Let $0<\varepsilon<(1 / 2)$. Choose a compact nowhere dense set $K \subseteq \mathbf{D}$, such that $(1 / \pi) \operatorname{Area}(\mathbf{D}-K)<\varepsilon$. Now, let $\Delta_{n}$ be the disks guaranteed by Lemma 2.2. Since the disks cluster exactly on the set $K$, we have $\operatorname{cl}\left(\bigcup_{n=1}^{\infty} \Delta_{n}\right)=$ $K \cup\left(\bigcup_{n=1}^{\infty} \operatorname{cl} \Delta_{n}\right)$. Also since the sum of the radii of $\Delta_{n}$ is finite, if we let $\mu$ be arc length measure on $\bigcup_{n=1}^{\infty} \partial \Delta_{n}$, then $S_{\mu}$ is pure. Clearly, $\sigma\left(S_{\mu}\right)=K \cup\left(\bigcup_{n=1}^{\infty} \operatorname{cl} \Delta_{n}\right)$ and $G=\operatorname{abpe}(\mu)=\bigcup_{n=1}^{\infty} \Delta_{n}$. Thus, $(1 / \pi) \operatorname{Area}\left[\sigma\left(S_{\mu}\right)\right] \geq(1 / \pi) \operatorname{Area}(K) \geq 1-\varepsilon$ and $\operatorname{tr}\left[S_{\mu}^{*}, S_{\mu}\right]=(1 / \pi) \operatorname{Area}(G)<\varepsilon$.

The next example shows that even if the essential spectrum of $S$ is small and the index function of $S$ is integrable, $S$ need not have trace class self-commutator. First a simple Lemma is needed.

Lemma 2.4. Suppose $\left\{K_{n}: n \in \mathbf{Z}^{+} \cup\{\infty\}\right\}$ is a sequence of disjoint compact sets such that the sets $\left\{K_{n}: n \geq 1\right\}$ cluster only on $K_{\infty}$. If $R\left(K_{n}\right)=C\left(K_{n}\right)$ for $n \in \mathbf{Z}^{+} \cup\{\infty\}$ and $K=\operatorname{cl}\left(\bigcup_{n=1}^{\infty} K_{n}\right)$, then $R(K)=C(K)$.

Proof. If $\mu$ is a measure on $K$ and $\mu \perp R(K)$, then since each $K_{n}, n<$ $\infty$, is isolated from the others including $K_{\infty}$, Runge's Theorem gives that the characteristic function of $K_{n}$ belongs to $R(K)$. Thus, if $f \in R\left(K_{n}\right)$ and $f$ is defined to be zero off $K_{n}$, then $f \in R(K)$. So, $\mu \mid K_{n} \perp R\left(K_{n}\right)$, thus $\mu \mid K_{n}=0$ for all $n<\infty$. Hence $\mu$ is supported on $K_{\infty}$. Now, if $U$ is any bounded component of $\mathbf{C}-K_{\infty}$, then $U-K \neq \varnothing$, else $U \subseteq \bigcup_{n=1}^{\infty} K_{n}$ and this contradicts the Baire Category Theorem. Hence Runge's Theorem says that $R(K) \mid K_{\infty}=R\left(K_{\infty}\right)$. Thus since $\mu$ is supported on $K_{\infty}$ and $R\left(K_{\infty}\right)=C\left(K_{\infty}\right)$ we see that $\mu=0$. Thus $R(K)=C(K)$.

It was shown by Hadwin and Nordgren, see [6], that a hyponormal operator $T$ whose essential spectrum has area zero, has trace class self-commutator if and only if its index function, $\operatorname{ind}(T-\lambda)$ is (area) integrable off $\sigma_{e}(T)$. We now show that a natural analog of this for subnormal operators is not true.

Example 2.5. There exists a pure subnormal operator $S$ with compact selfcommutator such that $R\left(\sigma_{e}(S)\right)=C\left(\sigma_{e}(S)\right)$, the index function, $\operatorname{ind}(S-\lambda)$, is integrable on $\sigma(S)-\sigma_{e}(S)$ and yet $\left[S^{*}, S\right]$ is not trace class.

Proof. To start, let $B_{0}=\mathbf{D}$ the unit disk and choose a totally disconnected compact set $K_{0}$ in $B_{0}$ with $(1 / \pi) \operatorname{Area}\left(B_{0}-K_{0}\right)<(1 / 2)$. Now, let $B_{n}, n \geq 1$, be the open disks guaranteed by Lemma 2.2. Also, let $r_{n}$, be the radius of $B_{n}$. Next choose positive integers $m_{n}$ such that $\sum_{n=0}^{\infty} m_{n} r_{n}^{2}=\infty$. For each $n \geq 1$, choose a totally disconnected compact set $K_{n} \subseteq B_{n}$ such that $\left(m_{n}+2\right) \operatorname{Area}\left(B_{n}-K_{n}\right) \leq$ $\left(1 / n^{2}\right)$. Next for each $n \geq 1$, apply Lemma 2.2 to get a sequence $\left\{\Delta_{n k}: k \geq 1\right\}$ of open disks in $B_{n}-K_{n}$ clustering exactly on $K_{n}$. Define for $n \geq 0, S_{n}=$ $M_{z}$ on $H^{2}\left(B_{n}\right)$ and for $n, k \geq 1$ define $S_{n k}=M_{z}$ on $H^{2}\left(\Delta_{n k}\right)$. Let $S=$ $\bigoplus_{n=o}^{\infty} S_{n}^{\left(m_{n}\right)} \oplus \bigoplus_{n, k=1}^{\infty} S_{n k}$. We claim that $S$ is the desired operator. Clearly $S$ 
is a pure subnormal operator. Also, if $K$ is compact and totally disconnected then $R(K)=C(K)$, hence Lemma 2.4 implies that $R\left(\sigma_{e}(S)\right)=C\left(\sigma_{e}(S)\right)$. Now, this last property guarantees that $S$ has compact self-commutator (see [1] or [5]). Also, if we set $T=\bigoplus_{n=0}^{\infty} S_{n}^{\left(m_{n}\right)}$, then $\operatorname{tr}\left[T^{*}, T\right]=\sum_{n=0}^{\infty} m_{n} r_{n}^{2}=\infty$. It follows that $T$ does not have trace class-self-commutator, hence $S$ cannot either. Finally, to show that the index function is integrable on $G=\sigma(S)-\sigma_{e}(S)$, it suffices to check this on the union $U$ of the disks $B_{n}$, because $|\operatorname{ind}(S-\lambda)| \leq 1$ for $\lambda$ not in $U$. Since $|\operatorname{ind}(S-\lambda)| \leq\left(m_{n}+2\right)$ for $\lambda \in B_{n}$ and $G \cap B_{n} \subseteq B_{n}-K_{n}$, we have

$$
\begin{aligned}
\int_{G \cap U}|\operatorname{ind}(S-\lambda)| d A & =\sum_{n=1}^{\infty} \int_{G \cap B_{n}}|\operatorname{ind}(S-\lambda)| d A \\
& \leq \sum_{n=1}^{\infty}\left(m_{n}+2\right) \operatorname{Area}\left(B_{n}-K_{n}\right)<\infty
\end{aligned}
$$

where the last sum is finite because $\left(m_{n}+2\right) \operatorname{Area}\left(B_{n}-K_{n}\right) \leq\left(1 / n^{2}\right)$.

In spite of the example above, there are other ways of measuring the "smallness" of a compact set. The following definition has proven useful in other settings. If $S$ is a subnormal operator and $K$ is a compact set in $\sigma(S)$, we say that $K$ is negligible (for the operator $S$ ) if the Toeplitz operator $S_{f}$ commutes with $S$ modulo the trace class operators, where $f$ is the Cauchy transform of the characteristic function of $K$.

Question. If $S$ is a pure subnormal operator such that $\sigma_{e}(S)$ is negligible and the index function of $S$ is integrable off $\sigma_{e}(S)$, then must $\left[S^{*}, S\right]$ be trace class?

3. Some generalizations. Theorem 1.3 applies to some rationally cyclic subnormal operators. Notice though that the region $G$ in Theorem 1.3 needs to be connected or have at most a finite number of components. Let $R^{2}(K, \mu)$ denote the $L^{2}(\mu)$ closure of the rational functions with poles off $K$.

Theorem 3.1. If $S=M_{z}$ on $R^{2}(K, \mu)$ is pure, $G=\operatorname{abpe}\left(R^{2}(K, \mu)\right)$ is connected and the reproducing kernels are dense in $R^{2}(K, \mu)$, then for $f \in$ $R^{2}(K, \mu) \cap L^{\infty}(\mu)$ we have $\operatorname{tr}\left[f(S)^{*}, f(S)\right] \geq(1 / \pi) \int_{G}\left|\hat{f}^{\prime}\right|^{2} d A$.

Proof. Since the reproducing kernels are dense, the natural map $R^{2}(K, \mu)$ $\rightarrow \operatorname{Hol}(G)$ allows us to identify $R^{2}(K, \mu)$ with a Hilbert space of analytic functions on $G$. Also, since $G$ is connected and $f(S)$ is a subnormal operator, so Theorem 1.3 applies.

Corollary 3.2. If $S=M_{z}$ on $R^{2}(K, \mu)$ is pure, $G=\operatorname{abpe}\left(R^{2}(K, \mu)\right)$ is connected and $\mathrm{cl} G=\sigma(S)$, then for $f \in R^{2}(K, \mu) \cap L^{\infty}(\mu)$ we have $\operatorname{tr}\left[f(S)^{*}, f(S)\right] \geq$ $(1 / \pi) \int_{G}\left|\hat{f}^{\prime}\right|^{2} d A$. 
Proof. Since $G$ is connected and $\operatorname{cl} G=\sigma(S)$, we have $\sigma_{e}(S)=\partial G$ and thus $R\left(\sigma_{e}(S)\right)=C\left(\sigma_{e}(S)\right)$. Now, if $\mathcal{M}$ is the collection of all functions in $R^{2}(K, \mu)$ that are orthogonal to the reproducing kernels, then $\mathcal{M}$ is a closed invariant subspace for $S$ and $\sigma(S \mid \mathcal{M}) \subseteq \sigma_{e}(S)$. Thus, since $R\left(\sigma_{e}(S)\right)=C\left(\sigma_{e}(S)\right)$, this implies that $S \mid \mathcal{M}$ is normal. Since $S$ is pure, $\mathcal{M}=(0)$ and the reproducing kernels are dense. Hence Theorem 3.1 applies.

The Berger-Shaw Theorem together with Corollary 3.2 gives the following result.

Corollary 3.3. If $S=M_{z}$ on $R^{2}(K, \mu)$ is pure, $G=$ abpe $\left(R^{2}(K, \mu)\right)$ is connected $\mathrm{cl} G=\sigma(S)$ then $(1 / \pi) \operatorname{Area}(G) \leq \operatorname{tr}\left[S^{*}, S\right] \leq(1 / \pi) \operatorname{Area}[\sigma(S)]$.

Corollary 3.4. If $S=M_{z}$ on $R^{2}(K, \mu)$ is pure, $G=\operatorname{abpe}\left(R^{2}(K, \mu)\right)$ is connected, $\operatorname{cl} G=\sigma(S)$ and Area $[\partial G]=0$, then $\operatorname{tr}\left[S^{*}, S\right]=(1 / \pi) \operatorname{Area}(G)$.

If $S=M_{z}$ on $R^{2}(K, \mu)$, then it is known that abpe $\left(R^{2}(K, \mu)\right)=\sigma(S)-$ $\sigma_{e}(S)$. Exactly when $\sigma_{e}(S)$ contributes to the trace of $\left[S^{*}, S\right]$ is unknown. For cyclic subnormal operators we have shown above that it does not. We now construct a pure rationally cyclic subnormal operator with $G=a b p e\left(R^{2}(K, \mu)\right)$ dense in $\sigma(S)$, yet $\sigma_{e}(S)$ has positive area and is part of the trace of $\left[S^{*}, S\right]$. That is, the principal function $g_{s}$ for $S$, is not zero a.e. on $\sigma_{e}(S)$.

Example 3.5. There exists a pure rationally cyclic subnormal operator $S$ with $G=\operatorname{abpe}\left(R^{2}(K, \mu)\right)$ dense in $\sigma(S)$, and yet $(1 / \pi)$ Area $[G]<\operatorname{tr}\left[S^{*}, S\right]<$ $(1 / \pi) \operatorname{Area}[\sigma(S)]$.

Proof. Suppose $L$ is a swiss cheese; that is, there are open disks $\Delta_{n}$ inside the unit disk $\mathbf{D}$ whose union is dense in $\mathbf{D}$ and with summable radii such that $L=\operatorname{clD}-\bigcup_{n=1}^{\infty} \Delta_{n}$. Let $\nu_{1}$ be arc length measure on $\bigcup_{n=1}^{\infty} \partial \Delta_{n}$. Now, let $B_{n}$ be an open disk with the same center as $\Delta_{n}$ but with half the radius. Also, let $\nu_{2}$ be arc length measure on $\bigcup_{n=1}^{\infty} \partial B_{n}$. If we set $K=L \cup \bigcup_{n=1}^{\infty} \operatorname{cl} B_{n}$ and $\mu=\nu_{1}+\nu_{2}$, then $S=M_{z}$ on $R^{2}(K, \mu)$ is a pure rationally cyclic subnormal operator. Further, $\sigma(S)=K, \sigma_{e}(S)=L \cup \bigcup_{n=1}^{\infty} \partial B_{n}$ and $G=\operatorname{abpe}\left(R^{2}(K, \mu)\right)=\bigcup_{n=1}^{\infty} B_{n}$. Now, if $S_{i}=M_{z}$ on $R^{2}\left(K, \nu_{i}\right)$, then $S=S_{1} \oplus S_{2}$. Since $S_{2}$ is a direct sum of functions of the unilateral shift, Lemma 1.1 applies to give that $\operatorname{tr}\left[S_{2}^{*}, S_{2}\right]=(1 / \pi) \operatorname{Area}(G)$. Also since $S_{1}$ is pure, $\operatorname{tr}\left[S_{1}^{*}, S_{1}\right]>0$. Thus $\operatorname{tr}\left[S^{*}, S\right]=\operatorname{tr}\left[S_{1}^{*}, S_{1}\right]+\operatorname{tr}\left[S_{2}^{*}, S_{2}\right]=$ $\operatorname{tr}\left[S_{1}^{*}, S_{1}\right]+(1 / \pi) \operatorname{Area}(G)>(1 / \pi) \operatorname{Area}(G)$.

Now simply direct sum $S$ with an operator $T$ from Example 2.1 or 2.3 whose spectrum is disjoint from $S$. This direct sum is the required rationally cyclic subnormal operator.

The previous example, is one of the standard examples of an $R^{2}(K, \mu)$ space where the reproducing kernels are not dense.

We shall closes with a natural question. 
Question. If $S=M_{z}$ on $R^{2}(K, \mu)$ is a pure rationally cyclic subnormal operator, $G=\operatorname{abpe}\left(R^{2}(K, \mu)\right)$ and $R\left(\sigma_{e}(S)\right)=C\left(\sigma_{e}(S)\right)$, then is $\operatorname{tr}\left[S^{*}, S\right]=$ $(1 / \pi) \operatorname{Area}(G)$ ?

Notice that this hypothesis $R\left(\sigma_{e}(S)\right)=C\left(\sigma_{e}(S)\right)$ is satisfied by every irreducible cyclic subnormal operator, so this is a natural generalization to the rationally cyclic case. Also this hypothesis guarantees that the reproducing kernels are dense in $R^{2}(K, \mu)$. Further, the example mentioned above with $\operatorname{tr}\left[S^{*}, S\right]>(1 / \pi) \operatorname{Area}(G)$ does not satisfy $R\left(\sigma_{e}(S)\right)=C\left(\sigma_{e}(S)\right)$.

\section{REFERENCES}

[1] A. Aleman, Subnormal Operators with Compact Self Commutator, Manuscripta Math. 91 (1996), 353-367.

[2] J. Arazy, S. Fisher, S. Janson 8 J. Peetre, An identity for reproducing kernels in a planar domain and Hilbert-Schmidt Hankel operators, J. Reine Angew. Math. 406 (1990), 179-199.

[3] K. Clancey, Seminormal Operators, Springer-Verlag, New York, 1979.

[4] J. B. Conway, The Theory of Subnormal Operators, Amer. Math. Soc., Providence, RI, 1991.

[5] J. B. Conway \& N. S. Feldman, The Essential Self-Commutator of a Subnormal Operator, Proc. Amer. Math. Soc. 125 (1997), 243-244.

[6] D. Hadwin 8 E. Nordgren, Extensions of the Berger-Shaw Theorem, Proc. Amer. Math. Soc. 102 (1988), 517-525.

[7] S. Katok, Fuchsian Groups, Univ. of Chicago Press, Chicago, 1992.

[8] M. Martin \& M. Putinar, Lectures on Hyponormal Operators, Birkhauser, Boston, 1989.

[9] R. F. Olin \& L. YANG, The Commutant of Multiplication by $z$ on the closure of Polynomials in $L^{1}(\mu)$, J. Funct. Anal. 134 (1995), 297-320.

[10] W. F. Osgood, A Jordan Curve of Positive Area, Trans. Amer. Math. Soc. 4 (1903), $107-112$.

[11] H. Sagan, Space-Filling Curves, Springer-Verlag, New York, 1994.

[12] J. E. Thomson, Approximation in the Mean by Polynomials, Ann. Math 133 (1991), 477-507.

This paper was written while the author was a graduate student working under the direccion of Professor John B. Conway. He received partial support from NSF grant DMS-9401027.

Department of Mathematics

Michigan State University

East Lansing, Michigan 48824

Received: February 17th, 1997. 